\title{
Working on the front line
}

\author{
Author: Geraldine O'Hara ${ }^{\mathrm{A}}$
}

On 23 March 2014, the World Health Organization confirmed an outbreak of Ebola virus disease in Guinea. By August 2014, a delayed international response resulted in an unprecedented humanitarian emergency occurring in Guinea, Liberia and Sierra Leone. In this outbreak, over 26,000 people have been infected with Ebola virus and more than 10,000 have died. Médecins Sans Frontières led the emergency response on the ground treating over 5,000 confirmed cases of Ebola. This article reflects on challenges faced working in an Ebola treatment centre and what future research may offer.

KEYWORDS: Ebola, aid work, MSF, West Africa, Sierra Leone

\section{Introduction}

In December 2013, a two-year-old child in the Guéckédou district of Guinea developed a febrile illness with vomiting and black stools. His death occurred a few days later and similar cases began to be reported in the local area, characterised by fever, diarrhoea, vomiting and an exceptionally high mortality rate. An investigational team from the Guinean Ministry of Health and Médecins Sans Frontières was deployed on 18 March 2014 and identified the responsible agent as the Ebola virus. On 23 March 2014, the World Health Organization (WHO) confirmed an outbreak of Ebola virus in Guinea. By 31 March, cases were detected in Liberia and by early April, reported in Sierra Leone; molecular typing confirmed identical strains of the Zaire Ebola virus responsible in each country.

The scarcity of resources in West African healthcare systems meant they were easily overwhelmed. Lack of running water in some facilities hampered even the simplest infection control precautions. Other practical problems such as staff shortages; unavailability of safe transport vehicles for patients and corpses; incorrect triage; and limited availability of appropriate personal protective equipment (PPE); all contributed to the amplification of the outbreak, particularly when coupled with local, regional and international apathy.

Ebola virus is a non-segmented, negative-sense, singlestranded RNA virus. The virus is a member of the family

Author: Aacademic clinical lecturer, London School of Hygiene and Tropical Medicine, London, UK, and medical doctor, Médecins Sans Frontières, Ebola Treatment Centre, Kailahun, Sierra Leone
Filoviridae, taken from the Latin 'filum', meaning threadlike, based upon their filamentous structure. There are four recognised species of Ebola virus (Zaire, Sudan, Ivory Coast and Bundibugyo) causing human disease, although Ivory Coast Ebola virus has only caused a single human fatality. A fifth species, the Reston Ebola virus is recognised to cause disease in primates; humans show evidence of seroconversion and therefore can be infected, however, there are no clinical manifestations. Since their discovery in 1967 there have been 20 recognised Ebola virus outbreaks, the majority of which have been confined to Central Africa (Sudan, Democratic Republic of Congo and Uganda notably) and have been caused by the Zaire Ebola virus, a particularly virulent species with case fatality rates in several outbreaks between $80-90 \%$. To date, outbreaks have involved less than 400 cases. The current outbreak represents a fifty-fold increase.

The Ebola virus is transmitted by direct contact with body fluids (blood, vomit, faeces, urine, semen, breast milk and sweat) of a symptomatic case; aerosol transmission has not been shown to occur outside of laboratory experiments although common sense dictates droplets contaminated with bodily fluids are strongly likely to be infective. Transmission to healthcare workers (HCWs) and amplification of outbreaks in healthcare facilities is a well recognised phenomenon in Ebola outbreaks, as is infection occurring during burial rite or handling bodies post-mortem. Infected individuals are not infectious themselves until they are symptomatic and the incubation period is $2-21$ days.

\section{Epidemiological and clinical features}

Symptoms of Ebola virus disease (EVD) are non-specific, with patients presenting with fever, malaise, fatigue, myalgia and arthralgia: all symptoms easily suggestive of diagnoses such as malaria or typhoid, which are common illnesses in West Africa. As the disease progresses, diarrhoea and vomiting develops between days 3-5. Fluid loss may be severe, up to $10 \mathrm{~L}$ per day in some reports, exacerbated by severe vomiting preventing any oral rehydration. ${ }^{1,2}$ Dehydration may become significant causing intravascular volume depletion and severe asthenia; headache, conjunctival injection, chest pain, abdominal pain, hiccups and delirium may accompany it. This gastrointestinal phase of illness lasts up to 10 days and development of multiorgan dysfunction leading to death may occur during this period. Once past 12-14 days the chance of survival greatly increases, although late deaths in presumed convalescent patients are recognised. 
Notably, in this particular outbreak, haemorrhage has not been a prominent feature and rarely is present upon presentation, prompting use of the term EVD, as opposed to haemorrhagic fever.

In this outbreak EVD has occurred equally in men and women. HCW are disproportionately affected; in Sierra Leone for example a total of 199 (5.2\%) of the total of 3,854 laboratory-confirmed Ebola cases reported by September 2014 were HCWs, representing a much higher estimated cumulative incidence of confirmed Ebola in the healthcare workforce compared with the general population. ${ }^{3} \mathrm{HCW}$ are at particular risk in underprepared countries at the beginning of an outbreak, where infection control policy may either not be in place or may be staggeringly under-resourced.

Published case fatality rates range between $31 \%$ and $70.8 \%$ in West Africa; outside of West Africa, evacuated patients cared for in critical care facilities exhibit a case fatality rate of approximately $10 \% .^{4-10}$

Poor prognostic factors include older age ( $>45$ years), high viral load at presentation (10 million EBOV copies per $\mathrm{mL}$ ), haemorrhage (particularly gingival, nasal and vaginal bleeding) and hiccups (personal observation). ${ }^{7,10}$

As alluded to earlier, late deaths during the recovery phase were observed to occur and were particularly disheartening to medical teams. Cardiac arrhythmias (as a result of electrolyte imbalances), myocarditis, pericardial effusions, encephalitis or venous thromboembolic disease may be responsible.

\section{Challenges}

Clinical

Clinical care provided many challenges, from dehydration in the staff, to lack of availability of point of care testing to guide intravenous fluid therapy. Sheer numbers also affected the ability of clinical teams in EVD management centres to provide the highest level of care. Ambient temperatures during the day time in West Africa were frequently in excess of $30^{\circ} \mathrm{C}$, meaning staff dressed in PPE had only limited time to spend in the isolation area due to risk of overheating, heat stroke and dehydration. Visits to the isolation area were limited to 60 minutes per individual and during this time all tasks had to be completed from washing and feeding people, to assessing and administering drugs to intravenous cannulation. In some centres at the height of the outbreak, patients outnumbered staff by a ratio of $10: 1$, this meant ensuring basic human needs were met adequately was a significant challenge.

It is vital to acknowledge that the predominant clinical syndrome in EVD is not haemorrhage but a triad of gastrointestinal symptoms - nausea, vomiting, and diarrhoea causing fluid loss, subsequent intravascular volume depletion and metabolic abnormalities. Several novel therapies have been trialled for EVD, often on a compassionate basis on evacuated medical staff, and shown promise, but as yet there is no proven EVD-specific therapy. The cornerstone of EVD management is supportive care - preventing the development of hypovolemic shock and consequent complications through adequate rehydration.

Intensity of care varied hugely between different centres in West Africa. As an example, an excellent British Army run treatment centre in Sierra Leone was able to provide biochemical and haematological analysis, pulse oximetry and central venous cannulation if required. This 12-bedded centre was designed to provide intensive medical care for infected HCWs, either as a bridge to recovery or prior to evacuation. ${ }^{11,12}$ Larger treatments centres, such as the 120-bedded ELWA 3 situated in Monrovia, Liberia, were only able to offer short-term intravenous therapy due to massive caseloads, limited number of HCWs and limited time in PPE. ${ }^{8,13}$ Protection of staff must remain a priority in high-risk situations.

To date, case fatality rates in individuals evacuated to the UK, US and Europe have been low, reflecting the intensity of treatment offered to patients, including renal replacement therapy, intubation and ventilation, and lectin affinity plasmapheresis, in addition to intensive haemodynamic and haematological/biochemical monitoring. ${ }^{1,2,14,15}$ Patients evacuated to hospitals in Europe or the US are more likely to be offered experimental therapies such as Zmapp, Zmab, favipiravir, FX06 and serum from convalescent survivors.

Admission of patients to treatment centres involved assessment of severity of illness using basic clinical tools level of consciousness or coma, quality of pulses, peripheral perfusion oliguria or anuria, and tachypnea. Use of PPE results in limited examination skills, impaired communication skills, often with removal of non-verbal skills, and limited time to perform assessments. Broadly speaking, rapid clinical assessment allowed triage of patients into one of three categories: those who are clinically hypovolemic, not in shock, and able to provide self-care; those who are hypovolemic, not in shock, but unable to self-care; and those in shock with evidence of organ failure.

Several centres prioritised oral rehydration with supporting antiemetics and antidiarrhoeals, often in desperate situations where staff struggled to provide minimal care. Intravenous fluids were reserved for hypovolemic but not shocked patients who were often suffering from asthenia and unable to eat or drink due to weakness. It must be acknowledged that establishing intravenous access, management of sharps, and delivering safe and effective volumes of fluid required intensive individual-level patient care, and careful selection of the cohort most likely to benefit was required. In general, these patients were given 48-72 hours of intravenous fluid as a bridge to reestablishing oral rehydration.

Treatment centres with the capacity to examine blood samples for malaria performed testing; in many centres however, treatment for malaria was administered as routine to minimise venepuncture and laboratory work, and consequent risk to staff. Broad-spectrum antibiotics, such as cefixime or ceftriaxone, were administered during the gastrointestinal phase of the illness in the belief that the effect of bacterial gut translocation could be minimised as well as any intercurrent infections treated.

In patients with clear evidence of terminal disease, symptom control with mouth care, positioning, and narcotics and benzodiazepines (if required) offer best end-of-life care. In the pursuit of aggressive treatment, it is vital not to forget management of the terminally ill.

Recording of simple clinical data was limited by an inability to take any material out of the isolation area, and in some centres, by limited electricity to power electronic data capture. Several 
centres had such low lighting at night that it was not possible to provide any clinical care due to the risks to staff.

Better characterisation of biochemical abnormalities would help to direct care for future patients in terms of fluid resuscitation. Measurement of haematological and coagulation parameters may also be of interest, but realistically, in resource-poor settings, treatment options for deranged clotting or low haemoglobin may be non existent; instead the addition or removal of potassium from fluids may prove a simple and life-saving intervention. Point-of-care testing appeals, but realistically is unfeasible with isolation areas due to high temperatures and dehydration of HCWs. However, portable basic biochemical analysers are available and could be incorporated into mobile virology laboratories which provided excellent diagnostics in West Africa during this outbreak.

\section{Special cases}

Patients themselves provided clinical challenges, in particular, pregnant women, recovering patients and children.

There is a large crossover between the presentation of women with pregnancy complications and the alert symptoms for a suspected Ebola case such as abdominal pain, chest pain, arthralgia, vomiting, vaginal bleeding, spontaneous miscarriage, stillbirth/intrauterine fetal death (IUFD) and fever. ${ }^{16}$ Intervention in obstetric crises such as these, without adequate testing and PPE, means nosocomial transmission may occur. Furthermore, during this outbreak we found that pregnant women with EVD provide their own management challenge. Prior to this outbreak, relatively little was known about the maternal and fetal outcomes in pregnant women infected with Ebola, except that mortality in pregnant women appeared higher than in non-pregnant women. In this outbreak, spontaneous miscarriage was observed and IUFD appeared to be inevitable, however women did survive. It is most likely that massive infection of the fetus occurs through the placenta. Furthermore, a mother with no detectable Ebola virus in her blood may still harbor viable virus in the amniotic fluid, placenta and indeed the fetus (personal observation). ${ }^{17}$ This raises the issue that 'cured' pregnant women cannot be discharged safely into the community and instead must undergo either a spontaneous miscarriage or assisted delivery in the treatment centre due to the potential for onward household or nosocomial transmission.

The risk to EVD in children is attributed to contact with their sick family members. Spread through breastfeeding has also been described, with Ebola virus detected in breast milk 15 days after disease in a lactating mother with a negative peripheral blood RT-PCR for Ebola. ${ }^{18,19}$ This discordance between body fluids needs rapid and adequate resolution to determine what advice to offer to lactating women regarding risk.

At present, it is unclear how long infective Ebola can be present in other body fluids, such as saliva, tears, urine, stool, breast milk, vaginal and amniotic fluid and seminal fluids. ${ }^{20} \mathrm{~A}$ patient treated in an isolation facility in Hamburg, Germany, had infective Ebola isolated from urine samples on day 26 of his illness, nine days after the clearance of virus from plasma. ${ }^{1}$ Ebola virus has been detected by molecular techniques in semen 101 days post illness and in a single vaginal sample at
33 days. This has led to the current public health advice to use condoms during any sexual intercourse for six months after onset of symptoms. However, this advice is based on findings from just 13 patients. ${ }^{18,21}$ In the post-outbreak setting, infectivity of surviving patients must be examined in order to build a robust body of evidence.

Little is known about the longer-term complications of Ebola; a study in Kikwit, Democratic Republic of the Congo, showed that in the first six months of follow-up, individuals recovering from Ebola reported arthralgia, myalgia, abdominal pain, extreme fatigue and anorexia more frequently than household contacts who had not been infected. ${ }^{22}$ There is a dearth of literature on the longer term psychological effects.

Unaccompanied children in treatment centres provide management dilemmas of their own. Unable to advocate for themselves or recognise needs for fluids, special attention is required to ensure they do not become dehydrated. On several occasions, convalescent teenage girls who remained in the centre awaiting discharge could be persuaded to take over management of younger children. Provision of intravenous fluids needs to be carefully considered especially in small children, as the nature of treatment centres means constant monitoring is unavailable. Consideration must also be given to the psychological effects a treatment centre may havefear, isolation and witnessing death, including deaths of loved ones - and several centres have trained local staff to work as counsellors and play therapists with children in the isolation area. On balance, specialist paediatric treatment centres providing paediatric expertise and a more intensive nurse-to-patient ratio would likely improve outcomes in this population.

\section{The future}

Several experimental drugs are being considered for trial in Ebola and the WHO has prioritised Ebola convalescent whole blood and convalescent plasma transfusion for evaluation. Investigations into the efficacy of humanised monoclonal antibodies, interfering RNA drugs and viral RNA polymerase inhibitor therapies are ongoing.

During this outbreak, an unprecedented decision was made to try and conduct a clinical trial of two experimental medications favipiravir and brincidofivir - in treatment centres in Liberia and Guinea. Novel trial designs were used to maximise the chance of detecting a significant effect while offering treatment to all. ${ }^{23}$ This represents a sea change in clinical research and is a clear acknowledgement of the seriousness of the outbreak. Trials of several different prototypical vaccines are ongoing and appear initially to be safe and immunogenic. ${ }^{24,25}$ However, many research questions, such as whether to use potassiumcontaining intravenous fluids, if loperamide is a safe way of reducing gastrointestinal losses, how long the virus persists in bodily secretions, and many others, remain unaddressed.

The prevention of spread of Ebola in Mali, Nigeria and Senegal shows that relatively simple measures, such as early identification, isolation, contact tracing and public engagement, can be used to prevent outbreaks developing. As cases appear to decline in West Africa, the international community must remain vigilant and not pin hopes on experimental therapies or as yet, untested vaccines. 


\section{Conclusions}

Outbreaks of infectious diseases with high mortality rates will be challenging in any context, but in resource-poor settings even simple tasks can prove almost insurmountable. Although difficult to perform, basic nursing care and, in particular, palliative care should not be forgotten. Staff safety in healthcare facilities must be a priority to protect HCW and reduce onward transmission; however when a centre is adequately staffed, intravenous cannulation and relatively complex interventions, such as central line insertion, are viable and will alter mortality rates.

\section{References}

1 Kreuels B, Wichmann D, Emmerich P et al. A case of severe Ebola virus infection complicated by gram-negative septicemia. $N$ Engl J Med 2014;371:2394-401.

2 Lyon GM, Mehta AK, Varkey JB et al. Clinical care of two patients with Ebola virus disease in the United States. $N$ Engl J Med 2014;371:2402-9.

3 Kilmarx PH, Clarke KR, Dietz PM et al. Ebola virus disease in health care workers - Sierra Leone, 2014. MMWR Morb Mortal Wkly Rep 2014;63:1168-71.

4 Schieffelin JS, Shaffer JG, Goba A et al. Clinical illness and outcomes in patients with Ebola in Sierra Leone. N Engl J Med 2014;371:2092-100.

5 Barry M, Touré A, Traoré FA et al. Clinical predictor of mortality in patients with Ebola virus disease. Clin Infect Dis 2015;60:1821-4.

6 Bah EI, Lamah M-C, Fletcher T et al. Clinical presentation of patients with Ebola virus disease in Conakry, Guinea. $N$ Engl J Med 2015;372:40-7.

7 Barry M, Traoré FA, Sako FB et al. Ebola outbreak in Conakry, Guinea: epidemiological, clinical, and outcome features. Med Mal Infect 2014;44:491-4.

8 Dallatomasina S, Crestani R, Sylvester Squire J et al. Ebola outbreak in rural West Africa: epidemiology, clinical features and outcomes. Trop Med Int Health 2015;20:448-54.

9 Ansumana R, Jacobsen K, Sahr F. Ebola in Freetown Area, Sierra Leone - a case study of 581 patients. N Engl J Med 2015;372:584-7.

10 WHO Ebola response team. Ebola virus disease in West Africa - the first 9 months of the epidemic and forward projections. $N$ Engl $J$ Med 2014;371:1481-95.

11 Rees PS, Lamb LE, Nicholson-Roberts TC et al. Safety and feasibility of a strategy of early central venous catheter insertion in a deployed UK military Ebola virus disease treatment unit. Intensive Care Med 2015;41:735-43.
12 Fowler RA, Fletcher T, Fischer WA II et al. Caring for critically ill patients with Ebola virus disease. Perspectives from West Africa. Am J Respir Crit Care Med 2014;190:733-7.

13 Chertow DS, Kleine C, Edwards JK et al. Ebola virus disease in West Africa - clinical manifestations and management. $N$ Engl J Med 2014;371:2052-4.

14 Büttner S, Koch B, Dolnik O et al. Extracorporeal virus elimination for the treatment of severe Ebola virus disease - first experience with lectin affinity plasmapheresis. Blood Purif 2014;38:286-91.

15 Connor MJ, Kraft C, Mehta AK et al. Successful delivery of RRT in Ebola virus disease. J Am Soc Nephrol 2014;26:31-7.

16 Black BO. Obstetrics in the time of Ebola: challenges and dilemmas in providing lifesaving care during a deadly epidemic. BJOG 2014; 122:284-6.

17 Baggi FM, Taybi A, Kurth A et al. Management of pregnant women infected with Ebola virus in a treatment centre in Guinea, June 2014. Euro Surveill 2014;19:20983.

18 Sonnenberg P, Field N. Sexual and mother-to-child transmission of Ebola virus in the postconvalescent period. Clin Infect Dis 2015; 60:974-5.

19 Moreau M, Spencer C, Gozalbes J et al. Lactating mothers infected with Ebola virus: EBOV RT-PCR of blood only may be insufficient. Euro Surveill 2015;20:21017.

20 Bausch DG, Towner JS, Dowell SF et al. Assessment of the risk of Ebola virus transmission from bodily fluids and fomites. J Infect Dis 2007;196:S142-7.

21 Mackay IM, Arden KE. Ebola virus in the semen of convalescent men. Lancet Infect Dis 2015;15:149-50.

22 Rowe AK, Bertolli J, Khan AS et al. Clinical, virologic, and immunologic follow-up of convalescent Ebola hemorrhagic fever patients and their household contacts, Kikwit, Democratic Republic of the Congo. Commission de Lutte contre les Epidémies à Kikwit. J Infect Dis 1999;179 Suppl 1:S28-35.

23 Cooper BS, Boni MF, Pan-ngum W et al. Evaluating clinical trial designs for investigational treatments of Ebola virus disease. PLoS Med 2015;12:e1001815.

24 Rampling T, Ewer K, Bowyer G et al. A monovalent chimpanzee adenovirus Ebola vaccine - preliminary report. N Engl J Med 2015 Jan 28, epub ahead of print.

25 Qiu X, Wong G, Audet J et al. Reversion of advanced Ebola virus disease in nonhuman primates with ZMapp. Nature 2014; 514:47-53.

Address for correspondence: Dr G O'Hara, Department of Clinical Research, London School of Hygiene and Tropical Medicine, Keppel Street, London WC1E 7HT, UK.

Email: drgeraldineohara@gmail.com 